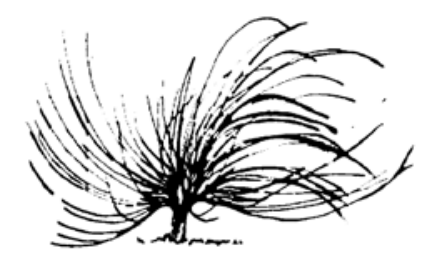

\title{
Reflexiones sobre la formación de competencias transversales y digitales en las instituciones de educación superior de México
}

\author{
Uzziel Japhet Vega Cadena ${ }^{1}$ \\ Universidad de Quintana Roo \\ Playa del Carmen, México \\ uzzielvc@uqroo.edu.mx
}

\author{
Mariana Figueroa de la Fuente ${ }^{2}$ \\ Universidad de Quintana Roo \\ Playa del Carmen, México \\ mfigueroa@uqroo.edu.mx
}

\author{
Francisco del Real Medina ${ }^{3}$ \\ Universidad de Quintana Roo \\ Playa del Carmen, México \\ fdelreal@uqroo.edu.mx
}

\section{Resumen}

La educación en el siglo XXI enfrenta el gran reto de formar profesionales que puedan encarar las necesidades laborales contemporáneas en un entorno globalizado y altamente tecnificado. Por lo que se requiere que la educación superior fomente el desarrollo de competencias laborales y digitales que permitan trabajar

\section{(C) (1) (3) $\Theta$}

http://dx.doi.org/10.15359/rep.12-1.1

Recibido: 11 de octubre de 2016-Aprobado: 9 de mayo de 2017

1 Maestro en Mercadotecnia, Maestro en Administración, Licenciado en Comunicación por el Tecnológico de Monterrey, Profesor investigador en la Universidad de Quintara Roo en Playa del Carmen, México.

2 Especialista en Gestión e Innovación en la Industria Gastronómica por Universidad del Caribe; Especialista en Gestión, Mercadotecnia y Artes de Mesa, Fundation Turquois, Mónaco, Maestra en Tecnología Educativa del Tecnológico de Monterrey, México. Profesora investigadora de la Universidad Quintana Roo en Playa del Carmen, México.

3 Maestro en Economía y Administración Pública por la Universidad de Quintana Roo; Licenciado en Contaduría por la UNAM; profesor investigador en la Universidad de Quintana Roo en Playa del Carmen, México. 
proyectos transversales e interrelacionados, para que el estudiantado tenga las herramientas necesarias para afrontar los cambios de la sociedad del conocimiento de forma competitiva. Por tanto, el objetivo del presente ensayo es reflexionar acerca del papel de la educación superior en México en la llamada sociedad del conocimiento, así como contribuir al análisis de las relaciones de las instituciones educativas con el Estado, la sociedad y el sector productivo. Para ello, en un primer apartado se abordan, de manera general, las políticas educativas vigentes en México; en un segundo apartado se realiza un acercamiento teórico a la definición de competencias; para finalmente revisar la importancia de las competencias digitales en la era actual. Por último, se presentan las conclusiones.

Palabras clave: competencias digitales, competitividad económica, educación superior, enseñanza, formación profesional.

\begin{abstract}
Education in the 21st century faces the great challenge of training professionals who can meet contemporary labor needs in a globalized and highly-skilled environment. Therefore, it is required that Higher Education foster the development of work and digital skills that allow crosscutting and interrelated projects to be developed so that the student has the necessary tools to deal with changes in the knowledge society in a competitive way. Therefore, the aim of this essay is to reflect on the role of higher education in Mexico in the so called "knowledge society" and contribute to the analysis of the relationship between educational institutions with the government, society, and productive sector. To this end, in a first section current Mexico's educational policies are described; in a second section, a theoretical approach to the definition of competencies or skills is made to finally review the importance of Digital Skills in the current age; finally, the conclusions are presented.
\end{abstract}


Keywords: digital skills, economic competitiveness, higher education, teaching, vocational training

\section{Introducción}

$\mathrm{D}$ urante los primeros tres años de la actual administración federal en México (2012-2018), la Presidencia de la República se fijó, como propósito, la realización de un conjunto de reformas estructurales ${ }^{4}$ para impulsar el desarrollo del país (Presidencia de la República, 2015), en un escenario complejo por los retos y desafíos existentes. Ente ellos, uno de los principales es el tema de la productividad, rubro que no ha tenido suficiente dinamismo debido a la crisis encarada por la nación y barreras, como las carencias en fortaleza institucional, desarrollo social, capital humano, igualdad de oportunidades y proyección internacional. Ello se ha manifestado en los últimos 30 años con un crecimiento negativo de la productividad, a una tasa promedio anual de $0.7 \%$, convirtiéndose en una de las principales limitantes para el desarrollo nacional (Secretaría de Gobernación, 2013).

En la actualidad, el reto es aún mayor, pues la mayoría de las reformas que se han formulado están en una etapa de implementación y el gobierno federal se encuentra ya en la segunda mitad del periodo de gestión. Las circunstancias de demora en la puesta en marcha de las reformas han hecho dudar a una parte de la población de los beneficios de estas y especular sobre sus efectos (Quintero, 2016).

Los diversos acontecimientos políticos en el país, tanto a nivel nacional como regional, entre los que se encuentran el marcado descenso en las estadísticas de aprobación de la Administración del Presidente Peña, la cual se aproxima al 22\%, que a la par de otros indicadores negativos como la percepción de la situación económica y la inseguridad, en los que el $63 \%$ de los sujetos encuestados a nivel nacional expresaron que no se ha tomado el camino correcto, el $46 \%$ que la economía ha empeorado, el $42 \%$ que se ha hecho un buen trabajo en salud y solo un $39 \%$ tiene una buena opinión respecto a la reforma en tema educativo (Parametría, 2016). Constituyen indicadores que ayudan a comprender los resultados negativos de las recientes elecciones intermedias, pues

4 Referido a modificaciones a todo el marco normativo en diversas materias, tales como: educación, energía y política. Inician modificando la constitución y en consecuencia las leyes y reglamentos secundarios relacionados. 
pueden ser considerados como síntomas de un profundo desencanto social, que obliga a las autoridades a acelerar el paso en la obtención de resultados a partir de la implementación de las reformas que ofrezcan cambios palpables entre la población, principalmente en áreas sensibles como la economía y la educación.

En el mismo orden de ideas, estudios como el realizado en los foros de consulta ciudadanas para dar forma al Plan Nacional de Desarrollo 2012-2018 demuestran que en la sociedad existe una percepción generalizada de que es necesario que el Estado ofrezca, por un lado, educación de calidad; y por el otro impulse el crecimiento económico a través del incremento de la productividad. Acciones que tienen el potencial de mejorar los niveles de bienestar de la población, al contribuir a alcanzar una economía más competitiva. Razón por lo que fueron tomadas en cuenta como pilares para diseñar y trazar los objetivos del Plan Nacional de Desarrollo 2012-2018 (PND). En este plan se considera la necesidad de realizar una reforma educativa y hacendaria para garantizar el desarrollo del país. Por tanto, para lograr estos objetivos, el Gobierno federal se ha propuesto desarrollar estrategias de reorientación del gasto hacia rubros o actividades que fortalezcan los factores productivos del país, así lo señaló el presidente Peña $(2014$, p. 29) al afirmar que: "Los ingresos que se obtengan de un recurso no renovable, como lo es el petróleo, se invertirán en nuevas formas generadoras de riqueza, como son precisamente la educación, la ciencia y tecnología, la infraestructura y el ahorro público".

Así, pues, el adecuado desempeño del Estado resulta fundamental para el desarrollo económico de un país, que con sus políticas públicas oriente el crecimiento económico. En el caso concreto de México, la Constitución Política de los Estados Unidos Mexicanos (CPEUM) otorga la rectoría del desarrollo nacional al Estado para que entre otras cosas, regule y fomente las actividades que demande el interés general (Artículo 25). Es así como, la reforma hacendaria se planteó después de un exhaustivo análisis del crecimiento económico y de los elementos necesarios para lograr que el país tenga una economía competitiva, así como de su profundo carácter social. En este sentido, de acuerdo con Videgaray (2014), el régimen fiscal debería constituir un elemento de redistribución de la riqueza al atender las necesidades básicas de la población, consagradas en la CPEUM, y con ello contribuir a lograr 
una educación de calidad ${ }^{5}$, aspecto que abona al fortalecimiento del Estado, ya que en la búsqueda de satisfacer los derechos sociales, este debería intervenir en la orientación del desarrollo integral del país, al garantizar el derecho a la educación y potencializar el desarrollo del capital humano.

Sin embargo, la relación entre la baja productividad del país y el desacelerado crecimiento económico se han vuelto temas recurrentes en el discurso planteado para justificar los pobres resultados en la implementación tanto de la reforma hacendaria como de la educativa, e incluso, de acuerdo con Videgaray (2014), es la razón por la que dentro del periodo 1990-2010 se dio un descenso aún mayor en la productividad de México. Tendencia que continua en la actualidad de acuerdo con los resultados ofrecidos por el Instituto Mexicano para la Competitividad (2014) que, mediante el indice de competitividad estatal 2014, evalúa las capacidades estructurales y coyunturales de las entidades, con el objetivo de medir la capacidad de las entidades federativas de atraer y retener talento e inversiones. Para ello se divide en 89 indicadores y 10 subíndices, cada uno de los cuales se encarga de evaluar una dimensión distinta de la competitividad. Y cuyos resultados señalan que las dimensiones que más han retrocedido son la de innovación y derecho, esta última debido a la crisis de violencia, además del incremento en la tasa de secuestros de 1.1 a 1.3 por cada 100 mil habitantes y la cantidad de periodistas que han matado o que han desaparecido pasaron de 45 a 66, factores que han hecho que la percepción de inseguridad vaya en aumento.

Con estos datos en mente, el Gobierno Federal argumenta que el reducido crecimiento de la productividad provoca como efecto un insuficiente dinamismo de la economía, por lo en la actual administración se busca, entre otros objetivos, el "democratizar la productividad" como una estrategia transversal, para elevar sus niveles y con ello mejorar el nivel de ingreso y de bienestar de los hogares en México. Por ello, en el mencionado PND 2013-2018 han quedado plasmadas tanto la estrategia de "democratizar la productividad" como la de un "México con educación de calidad" como una de las cinco metas nacionales trazadas de acuerdo con la tabla 1 .

5 Ver la reforma al artículo 3 de la Constitución Política de los Estados Unidos Mexicanos que señala que "El Estado garantizará la calidad en la educación obligatoria de manera que los materiales y métodos educativos, la organización escolar... garanticen el máximo logro de aprendizaje de los educandos". 


\section{Tabla 1: Objetivos, metas y estrategias del Plan Nacional de Desarrollo 2013-2018}

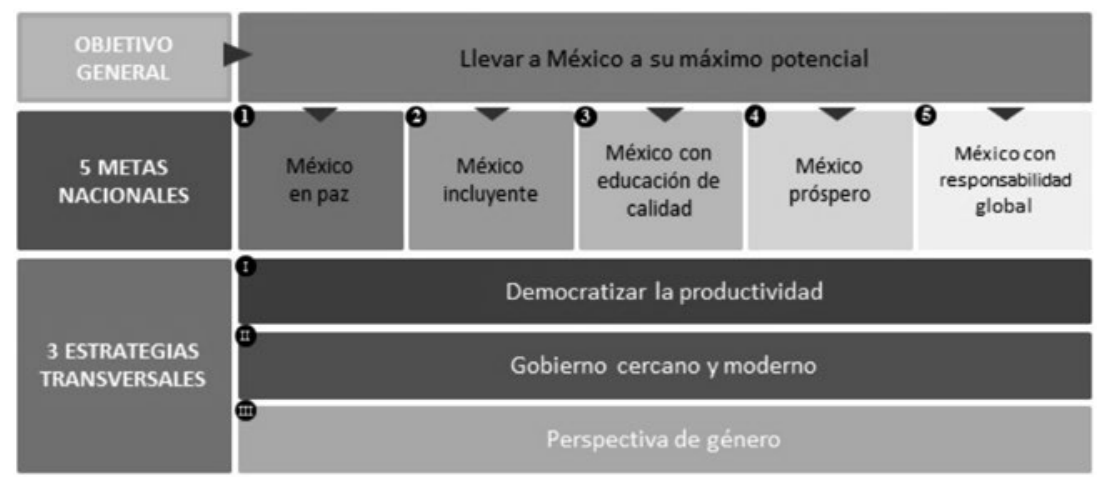

Nota: Plan Nacional de Desarrollo 2013-2018 (2013, p. 21).

Entre las cinco metas nacionales, la educación de calidad requiere del establecimiento de políticas públicas que enlacen el trabajo educativo con el conocimiento científico, el desarrollo tecnológico y la innovación para que los diversos sectores de la sociedad se modernicen generando con ello mayor valor por su trabajo. La reforma educativa busca, entonces, atender uno de los reclamos más añejos de la sociedad mexicana, mejorar la calidad de la educación, por lo que implica modificar el escenario actual en donde la enorme cantidad de recursos destinados a la educación no sean minimizados por los elevados índices de abandono escolar, rezago, o bien por infraestructura insuficiente o deteriorada, o peor aún, por un bajo nivel de desempeño académico del estudiantado en comparación con estándares internacionales.

Por otra parte, el modelo educativo 2016 (Secretaría de Educación Pública, 2016) ha sido elaborado con un enfoque humanista y partiendo de los avances de las ciencias de la educación, para reorganizar los principales componentes del sistema educativo nacional y poder, de esta forma, ofrecer una enseñanza integral a la altura de las exigencias del siglo XXI. En esta nueva visión la escuela se vuelve el centro del sistema educativo, lo que permite concretar el planteamiento pedagógico y asegurar el logro de los fines de la educación; cuestión que solo será posible con docentes con mejor preparación, capaces de transmitir aprendizajes significativos con creatividad e innovación y el uso de las tecnologías de la información y la comunicación (TIC) 
con fines educativos. Para lo cual "se plantea la consolidación de los procesos de evaluación y del Servicio Profesional Docente, así como el fortalecimiento de la formación inicial, entre maestros y estudiantes, para atraer y retener a los mejores docentes" (p. 19). Finalmente, este modelo educativo con principios intrínsecos de la tarea educativa, la inclusión y la equidad ha implantado mecanismos de colaboración entre el gobierno federal y los gobiernos estatales, así como entre la autoridad educativa y el sindicato de docentes, para asegurar el correcto funcionamiento del sistema educativo y una gobernanza efectiva.

Por lo anterior, y por la profundidad de las causas en las deficiencias de la educación en México, así como por lo complejo y ambicioso de la meta trazada, se deduce que tanto la reforma como la implementación en materia educativa son un proceso vivo, de largo plazo y al cual gobierno y sociedad en conjunto deben contribuir permanentemente. Factores que aunados a los fenómenos globales como el desarrollo tecnológico y los cambios vertiginosos en los medios de comunicación, demandan de la sociedad una competitividad creciente basada en fuertes incrementos de productividad, calidad e innovación, lo que a su vez exige al recurso humano incrementar sus habilidades por medio de la capacitación y sistemas de aprendizaje que le permitan garantizar un desarrollo permanente (Díaz y Arancibia, 2011). En este contexto, el enfoque de competencias surge como una forma de resolver tales demandas, ya que uno de sus objetivos primordiales es desarrollar ventajas competitivas reales en las personas, por lo que a continuación se revisa su significado y evolución.

\section{Enfoque de las competencias laborales}

Por medio de la educación se transmiten conocimientos, actitudes y valores que emanan de una filosofía que ha sido validada en un contexto social e histórico determinado. Por tanto, si se pretende estudiar la educación de manera científica, resulta necesario abordarla como parte innegable de la filosofía. Es así como la historia de la evolución de las distintas civilizaciones va íntimamente ligada a los cuestionamientos existenciales y del comportamiento de la realidad que el ser humano por naturaleza se ha planteado. Por ende, los cimientos de pensamiento y la educación actuales comenzaron su edificación desde la Grecia de los presocráticos, en donde comienza la sensibilidad artística y el interés 
por la inteligencia, preceptos de las distintas concepciones pedagógicas (Aguirre, 2010; Altarejos y Naval, 2011).

De forma tal, el proceso educativo emana de ideas filosóficas de manera directa o indirecta, concebidas en un tiempo y lugar determinado, influenciadas por convicciones políticas y religiosas previas que tratan de entender qué es el ser humano, cuál es su puesto en el mundo y determinar el progreso de la sociedad. Muy importante para determinar tales fines de la educación, han sido los cuestionamientos planteados acerca de cuál sea la más fundamental de las facultades en el ser humano u otras acerca de la naturaleza del individuo y su relación con la comunidad. Para ello, surgieron filosofías de la educación de sesgo intelectualista, emotivista, voluntarista, personalistas, individualistas, colectivistas, entre otras (Ferrater, 2003). Es decir, desde el origen del ser humano ha existido una transferencia continua de conocimientos y enseñanzas que han sobrepasado tiempo y espacio generacionalmente; las razones que detonan este fenómeno van desde tener un origen como respuesta para mantener la sobrevivencia, ser un eslabón más en el proceso evolutivo o una necesidad para mantener la especie y además para algunos, la transferencia fue la respuesta con un fin trascendental, que de acuerdo con Fromm (2012), contribuye al buen funcionamiento y desarrollo de la personalidad en el ser humano.

Independientemente del objetivo de la transferencia continua de conocimientos en la época contemporánea, dicha transmisión hace una importante aportación al desarrollo local y global, además de brindar continuidad y certidumbre para no cometer los mismos errores de antaño, el tener el conocimiento adecuando en el momento adecuando, por lo que es, entonces, hoy, una necesidad obligada, si se pretende ser lo óptimo como institución o individuo, inclusive, es un referente para alcanzar el éxito en el camino agreste y competitivo profesional actual. Existen modelos que hablan de la adquisición del conocimiento técnico y la forma como se desarrolla, pero la discusión más importante radica en saber cuál es el conocimiento que actualmente se requiere para impactar positivamente en el entorno profesional. En el caso de México, los esfuerzos gubernamentales de organismos como el Consejo Nacional de Normalización y Certificación de Competencias Laborales (2016) ${ }^{6}$

6 Conocido en México como el CONOCER “es la entidad del Gobierno Federal que promueve y coordina el Sistema Nacional de Competencias de las personas, para contribuir al crecimiento económico, el desarrollo educativo y el progreso social de México". 
aportan un referente del Gobierno Federal para indicar conocimientos técnicos o estándares de competencia ${ }^{7}$ que pueden fortalecerse para aportar competitividad económica y eventualmente desarrollo educativo y progreso social a México. La certificación del conocimiento pretende satisfacer las necesidades del entorno, pero el desarrollo de una actividad profesional no solo requiere de conocimiento técnico, debido a que el nuevo modelo productivo revalora la importancia del trabajo humano más allá del esfuerzo físico, enfocándose en la inteligencia, creatividad y en la capacidad de adaptación a los cambios; por ello, se requiere desarrollar un proceso de aprendizaje continuo a lo largo de la vida productiva (Ibarra, 2000).

De esta forma, el inculcar el conocimiento basado en actitudes ha progresado de la mano de la evolución educativa, como se puede ver en la revisión realizada por Von Schelling (2005), quien afirma que el sistema escolástico racionalista evolucionó al empirismo, ambos de igual de radicales; la educación requería un equilibrio y por supuesto la llegada del término medio y conciliar entre varias corrientes pedagógicas. La radicalidad racionalista demandaba la adquisición de conocimiento del mundo a través del pensamiento exclusivamente, sin participación de la experiencia. La radicalidad empírica, por su lado, afirmó que la experiencia forma el conocimiento; si bien este conflicto parecía interminable, ambas escuelas presentaban áreas de oportunidad. El racionalismo, al hablar de la formación de una idea o juicio del mundo sin experimentar, argumentaba sobre una verdad absoluta, pero que no parecía justificada, por tanto era necesario, entonces, experimentar para construir cualquier conocimiento adicional. Al tomar lo mejor de ambas corrientes se originó el idealismo trascendental.

Por otra parte, prácticamente desde su origen, las instituciones educativas han estado conscientes de que el conocimiento no solo puede o tiene que ser un ejercicio mental sino además requiere un complemento a través de un fortalecimiento físico y espiritual, el individuo requiere una serie de habilidades que le permitan ser, al egresar, más competitivo. Como señala Delors (2013), la educación a lo largo de la vida se basa en cuatro pilares: aprender a conocer, aprender a hacer, aprender a vivir juntos, aprender a ser. Por lo que cabe resaltar la

7 "Los Estándares de Competencia son las definiciones de los conocimientos, habilidades, destrezas y actitudes requeridas, para que una persona realice cualquier actividad productiva, social o de gobierno, con un nivel de alto desempeño, definidos por los propios sectores". 
importancia de desarrollar actitudes y valores personales, de la mano de conocimientos académicos, para que el estudiantado se desarrolle como seres humanos integrales.

Dichas características actitudinales y valores son conocidas como competencias transversales y van de la mano del conocimiento (Denyer, Poulain, y Vanloubbeeck, 2004). En este sentido, el concepto de competencia, según la Real Academia Española (2014), proviene del latín competentia que implica "oposición o rivalidad entre dos o más personas que aspiran a obtener la misma cosa", sin embargo, también la describe como "pericia, aptitud o idoneidad para hacer algo o intervenir en un asunto determinado".

En el mismo orden de ideas, para la Dirección General de Educación Superior para Profesionales de la Educación (2016), la competencia es el conjunto de capacidades constituidas que se forman con actitudes, habilidades, aptitudes y destrezas en un individuo mediante procesos de aprendizaje y que se manifiestan en situaciones y contextos diversos. Elllo concuerda con Spencer y Spencer (1993), quienes señalan que "una competencia es una característica subyacente de un individuo que está causalmente relacionada a un criterio referenciado como efectivo y/o un desempeño superior en un trabajo en una situación" (p. 10).

Asimismo, para la Organización para la Cooperación y el Desarrollo Económicos (2016), una competencia es la capacidad para responder a las exigencias individuales o sociales cada competencia tiene una combinación de habilidades prácticas y cognitivas que están relacionadas con los conocimientos, motivación, valores, actitudes, emociones y elementos sociales o comportamientos que actúan en su conjunto de manera eficaz. Por otra parte, Tobón (2008) define las competencias desde la línea del pensamiento complejo con seis aspectos esenciales: Procesos, complejidad, desempeño, idoneidad, metacognición y ética. Y hace referencia a que es un enfoque para la educación y no un modelo, porque solo se focalizan en ciertos aspectos conceptuales y metodológicos de la educación y la gestión del talento humano; pero no infieren los procesos instructivos, didácticos, ni conceptuales desde el punto de vista epistemológico, así como tampoco pretenden determinar la forma cómo debe ser el tipo de persona a formar. Por tanto, Tobón define las competencias de la siguiente forma: 
Procesos complejos de desempeño con idoneidad en determinados contextos, integrando diferentes saberes (saber ser, saber hacer, saber conocer y saber convivir), para realizar actividades y/o resolver problemas con sentido de reto, motivación, flexibilidad, creatividad, comprensión y emprendimiento, dentro de una perspectiva de procesamiento metacognitivo, mejoramiento continuo y compromiso ético, con la meta de contribuir al desarrollo personal, la construcción y afianzamiento del tejido social, la búsqueda continua del desarrollo económico-empresarial sostenible, y el cuidado y protección del ambiente y de las especies vivas. $(2008$, p. 5$)$

Y finalmente, parafraseando a Cazarez y Cuevas (2007), la competencia en el ámbito educativo establece una correlación de las habilidades cognitivas, psicológicas y motoras así como comportamientos afectivos y sociales, todo esto enfocado en las actividades, acciones y tareas específicas y personales.

Así, pues, la importancia de las competencias estaba basada en la especialización de los puestos de trabajo, pero a medida que la relación entre uno o varios procesos aumenta, las competencias especializadas de un puesto no son suficientes y se requiere tener otras que se relacionan con el trabajo en común, trabajar en proyectos transversales e interrelacionados con los diferentes equipos y áreas (Universitat Oberta de Catalunya, 2013). Existen, entonces, competencias que contienen los conocimientos técnicos específicos y teóricos propios de una especialización de una materia o disciplina, por otro lado también existen habilidades o competencias que son propias de la personalidad de cada individuo que lo complementan, distinguen y son inclusive rasgos únicos de su personalidad, estas últimas son las llamadas competencias genéricas o transversales que, complementadas con las competencias técnicas, forman un perfil en conjunto.

Al respecto, Cano (2008) lleva a cabo un desglose histórico de los conceptos de competencia y habla de la importancia de su evaluación dentro del marco educativo universitario, destacan elementos que permiten su comprensión y plantea que las competencias van más allá de lo aprendido en aula y del conocimiento teórico. Asimismo, da cuenta de que las competencias vinculan un meta conocimiento entre las actitudes, conceptos y procesos, es decir, permite que el individuo 
no solo acumule conocimiento, actitudes y procedimientos sino que seleccione cuáles acumular; todo esto, además, es vinculado con los rasgos de personalidad en un proceso continuo de aprendizaje, que forma la personalidad y la va forjando continuamente: nunca se es competente para siempre.

Por último, son González, Wagenaar, y Beneitone (2004) quienes afirman que las competencias implican tomar acción con reflexión, es decir, impactan dependiendo de cada situación y momento, siempre con un referente reflexivo que permita resolver situaciones efectivamente. Las competencias transversales son aquellas que se relacionan con el desarrollo personal sin que exista una dependencia de un ámbito temático o disciplinario específico, es decir, se encuentra en todos los dominios y acciones actitudinales, profesionales y académicas. Dichas competencias tienen un impacto directo en la contratación de recién egresados y egresadas de las universidades; pero también en la gente que tiene mayor experiencia, esta última puede ofrecer sus servicios a las organizaciones a través de su desempeño. Ambos casos benefician y maximizan las ganancias e indicadores de las organizaciones donde trabajan y es así donde el mercado hace la diferencia entre una candidatura y otra, lo que se refleja en el sueldo que perciben.

En este sentido, las competencias transversales tienen un gran peso y se convierten, así, en ventaja competitiva entre un individuo y otro; conforma un perfil adecuado para cada posición. La ventaja competitiva en toda la organización a través de su recurso humano es palpable inclusive si, a la larga, esta se convierte en parte de su cultura organizacional. Así pues, las competencias dentro de los programas académicos brindan una escala común global para determinar los perfiles de sus egresados y egresadas, los diferenciales que cada institución de educación superior integra en sus esquemas académicos no solo les hacen mejores, sino les sirve inclusive para la acreditación o mantener acreditaciones como prueba de la garantía que ofrecen al brindar los conocimientos adecuados y competitivos para sus prospectos posicionándoles mejor.

Al respecto, en el Espacio Europeo de Educación Superior (en Cano, 2008) se ha debatido sobre la importancia de las competencias transversales o genéricas precisamente en la formación del estudiantado titulado universitario, de esta forma se genera un marco contextual global tomando en cuenta la definición precisa de competencias que puede y debe esperarse del alumnado graduado en cada disciplina. Las competencias genéricas o 
transversales, ratificadas por la Comunidad Europea (Rodrigues, Peralta, y Nunes, 2011) son: 1. Analizar y sintetizar; 2. Aprender; 3. Resolver problemas; 4. Utilizar los conocimientos en la práctica; 5. Adaptarse a diferentes situaciones; 6. Preocuparse por la calidad; 7. Practicar las técnicas de la información y comunicación; 8. Trabajar de manera autónoma; 9. Trabajar en equipo; 10 . Organizarse y planificar.

Ahora bien, para finalizar, cabe destacar que en la actualidad la materia prima de la nueva economía es la información que se genera, comparte y produce de forma digital. Lo que Area (2010) llama la tercera revolución económica "la que está aconteciendo en el siglo XXI, basa sus productos en la transformación y reelaboración de información en sus múltiples formas para que sea comprada y consumida" (p. 2). Por tanto, señala que se necesita formar en competencias informacionales y digitales en la educación superior, con el objetivo de que las nuevas generaciones profesionistas puedan aprovechar las ventajas que ofrece el ecosistema informacional y tecnológico de la era actual e integrarse a sus procesos económicos, sociales y culturales; razón por la que se aborda este tema en el siguiente apartado.

\section{Competencias informacionales en la era digital}

En la era actual del conocimiento, los avances en las tecnologías de información y comunicación (TIC) han revolucionado los medios y soportes de distribución de la información, lo que ha permitido crear acceso a vastos volúmenes de datos; elevando, con ello, el grado de complejidad de las herramientas de comunicación y la selección de estas mismas. Dichos cambios han tenido repercusiones en los distintos ámbitos de comunicación e interacción del ser humano, alcanzando, sin duda, el plano educativo, ya que la manera en que el conocimiento se adquiere, produce y distribuye se ha hecho cambiante y efímero. Por tanto, se requiere adaptarse constantemente para ser capaz de interpretar los nuevos signos, iconos y textos propios de un nuevo contexto, canal y forma de comunicación, ya que el ejercicio mental requerido para descifrar e interpretar dichas tareas se hace de una forma completamente distinta a la que se usaba hasta ahora (Area, 2008).

En este escenario, para poder ser partícipes de los adelantos de las TIC, tener acceso a la información y educación de calidad, pero sobre todo para poder hacer uso adecuado de ellas, resulta imprescindible 
desarrollar capacidades básicas de adaptación y flexibilidad. A la par de ocho competencias específicas que, de acuerdo con O'Malley, Vavoula, Glew, Taylor, Sharples y Lefrere (2003), habilitan la posibilidad de lograrlo, las cuales son: resolución de problemas, trabajo colaborativo, creatividad e imaginación, comunicación, autoconciencia del conocimiento, autodirección y destrezas del aprendizaje, así como el dominio personal (ver tabla 2). Por otra parte, se debe complementar con el desarrollo de competencias transversales, entre las que destacan: la capacidad de buscar, filtrar, comprender, reproducir y compartir información digital de forma legal y ética (Ramírez, 2012).

\section{Tabla 2: Competencias específicas para aprender en la era del conocimiento}

\begin{tabular}{ll}
\hline Competencia & \multicolumn{1}{c}{ Descripción } \\
\hline $\begin{array}{l}\text { Resolución de } \\
\text { problemas }\end{array}$ & $\begin{array}{l}\text { Búsqueda de información, capacidad de toma de decisiones, } \\
\text { enmarcar problemas, hacer uso del pensamiento analítico y } \\
\text { conceptual. }\end{array}$ \\
\hline $\begin{array}{l}\text { Trabajo } \\
\text { colaborativo }\end{array}$ & $\begin{array}{l}\text { Capacidad de entablar empatía para construcción de } \\
\text { relaciones personales positivas, habilidad de persuasión } \\
\text { por medio de la argumentación lógica y racional; compartir } \\
\text { información para alcanzar metas. }\end{array}$ \\
\hline $\begin{array}{l}\text { Creatividad e } \\
\text { imaginación }\end{array}$ & Habilidad de buscar opciones y soluciones alternas. \\
\hline Comunicación & $\begin{array}{l}\text { Habilidad de escuchar atentamente y expresarse } \\
\text { correctamente de forma oral y escrita. }\end{array}$ \\
\hline $\begin{array}{l}\text { Autocontrol del } \\
\text { conocimiento }\end{array}$ & $\begin{array}{l}\text { Habilidad de adaptar el modelo mental a distintas } \\
\text { circunstancias; saber lidiar con presiones y emociones; } \\
\text { tomar la responsabilidad del propio aprendizaje y conocer } \\
\text { el estilo personal de aprendizaje. }\end{array}$ \\
\hline $\begin{array}{l}\text { Autodirección } \\
\text { del aprendizaje }\end{array}$ & $\begin{array}{l}\text { Capacidad de enfocarse en el logro de objetivos; recuperar, } \\
\text { analizar y sintetizar información, vincular y aplicar el } \\
\text { información. }\end{array}$ \\
\hline $\begin{array}{l}\text { Destrezas de } \\
\text { aprendizaje }\end{array}$ & $\begin{array}{l}\text { Comprensión del proceso de aprendizaje general y propio; } \\
\text { saber aprender a aprender. }\end{array}$ \\
\hline $\begin{array}{l}\text { Dominio } \\
\text { personal }\end{array}$ & $\begin{array}{l}\text { Habilidad de convertir las competencias en capacidades; } \\
\text { pomprensión del valor de las competencias; visión y valores } \\
\text { personales, ser realista. }\end{array}$ \\
\hline
\end{tabular}

Nota: O'Malley, Vavoula, Glew, Taylor, Sharples y Lefrere (2003). 
Asimismo, se requiere que los sujetos aprendices participen en proyectos formativos complejos por medio de la tecnología, que requieran enfocarse hacia resultados y fomenten el desarrollo de habilidades para el trabajo colaborativo, la gestión de recursos y la capacidad de investigar en la web. Ello les permitirá, en el largo plazo, desarrollar habilidades cognitivas complejas, como la capacidad de análisis, pensamiento crítico, comunicación asertiva, trabajo colaborativo, resolución de problemas y trabajar en tareas con recursos restringidos. De ahí la importancia de formar a las nuevas generaciones en ambientes innovadores que las confronten con la vida real, como fue señalado por la Organización para la Cooperación y Desarrollo Económicos (OCDE), que reafirma la necesidad de la incorporación al medio laboral de entes autónomos, críticos, competentes para la resolución de problemas y toma de decisiones de forma ética. Razones por las cuales resulta inminente la necesidad de que las instituciones de educación superior aseguren que las nuevas formas de instrucción con aplicación de herramientas digitales de comunicación permitan que sus estudiantes adquieran las competencias de alfabetización digital, gestión de proyectos habilidades de pensamiento crítico, sistémico, habilidades interpersonales y de autonomía (Ferrari, 2012), así como un elevado grado de apropiación tecnológica.

Por consiguiente, los procesos formativos requieren de esquemas innovadores y flexibles que deberán concebirse desde una visión multidisciplinar que permita el desarrollo de competencias para la generación, integración y socialización de información, que permitan profundizar el conocimiento (Ramírez, 2012). Esto puede lograrse concibiéndolo para tal efecto desde su diseño, en aspectos como la capacidad de adaptación a tiempos y ambientes distintos, que permitan asegurar el aprendizaje permanente, dar sentido de pertenencia estudiantil, además de aprovechar las ventajas que el movimiento e informalidad de los nuevos dispositivos tecnológicos ofrecen en el proceso de enseña aprendizaje (Naismith, Sharples, Vavoula y Lonsdale, 2004). Con esto el profesorado podría asegurar que está desarrollando en sus estudiantes las competencias digitales requeridas, para integrarse con mayor facilidad al medio laboral de la sociedad del conocimiento y con ello fortalecer la competitividad económica y eventualmente el desarrollo educativo y progreso social de México.

En este sentido, es importante mencionar que, si bien las ventajas de esta tendencia educativa están ampliamente reconocidas 
por organismos internacionales como la Organización de las Naciones Unidas para la Educación, la Ciencia y la Cultura (UNESCO en Didou, 2014), también es una realidad que se ha caído en algunos casos en prácticas comerciales sin sustento pedagógico, que van en detrimento de la calidad educativa que se busca, es decir, el riesgo es la mercantilización de la educación superior, con la proliferación de universidades internacionales de baja calidad. De ahí la importancia de regular la apertura de modelos institucionales que utilicen formas innovadoras de atención al alumnado, pero que contemplen mecanismos que les permitan velar por los objetivos académicos, para entonces sí poder obtener las ventajas que ofrecen las innovaciones tecnológicas en los indicadores educativos, como son: el incremento de la cobertura al impulsar la inclusión a la matrícula de grupos sociales vulnerables, dotarlos de saberes significativos, lograr disminuir la deserción, asegurar el egreso y empleabilidad, entre otros.

Desafortunadamente, en México aún se cuenta con importantes barreras como la brecha digital, ya que de 112 millones 336 mil habitantes, solo 51.2 millones usuan internet (Asociación Mexicana de Internet, 2014), por tanto, más de la mitad de la población no cuenta con la posibilidad de participar en la sociedad de la información y el conocimiento, lo cual convierte la brecha digital en uno de los principales problemas por resolver en el siglo XXI para México, pues constituye uno de los nuevos rostros de la desigualdad en el país (De la Selva, 2015). Además del hecho de que el país se encuentra dentro de las cinco naciones con el mayor déficit de habilidades para cubrir las necesidades de los grupos empleadores, debido a los rápidos avances tecnológicos y la digitalización del espacio de trabajo, según indican datos recientes de la Organización para la Cooperación y el Desarrollo Económicos, que señalan que el $45 \%$ del personal empleado tiene la percepción de que no cumple con el conjunto de habilidades apropiadas para desempeñarse en el trabajos eficazmente (World Economic Forum, 2016).

Y qué decir de los terribles resultados de las pruebas internacionales como los del Programa para la Evaluación Internacional de Alumnos (PISA, por sus siglas en inglés) que señalan que el 55\% de alumnado mexicano no alcanza el nivel de competencia básico en matemáticas, $41 \%$ en lectura y el $47 \%$ en ciencias (Organización para la Cooperación y el Desarrollo Económicos, 2012). Aunado a lo anterior, los docentes aún reportan la carencia de procesos y políticas que brinden soporte para 
trasformar la enseñanza, además de que no es común que la normatividad de las IES apoye la planeación adecuada del proceso de implantación de innovaciones curriculares (Díaz-Barriga, 2010). Por tanto, es pertinente señalar que el éxito en la innovación educativa dependerá, por una parte de la forma en la que los actores educativos operen los cambios, pero también por la forma en que estos sean regulados (Salinas, 2004), ya que es un proceso que afecta el desde el contexto micro como el salón de clases, hasta el conjunto macro de instituciones de educación superior y conlleva a su vez factores psicopedagógicos, culturales, ideológicos, económicos y políticos, de ahí que sea catalogado como un proceso multidimensionado (Kezar, 2001).

\section{Conclusiones}

En el actual escenario globalizado caracterizado por los cambios acelerados de la ciencia y tecnología, en especial el de las tecnologías de información y comunicación, las instituciones de educación superior deben atender las necesidades propias del desarrollo social, económico y ambiental del país en el que se ubican, por medio de estrategias de innovación y competitividad.

Es necesario que se sigan fortaleciendo las competencias pertinentes de acuerdo con las necesidades laborales de cada sector productivo para encarar los retos de la era actual, pero además hay aún trabajo pendiente para cerrar la brecha digital y fomentar el desarrollo de competencias digitales que permitan egresar, de las universidades, capital humano innovador, competitivo y altamente capacitado que contribuya a vencer los retos del desarrollo social y económico de México. Si bien los denominados sujetos nativos digitales hacen uso cotidianamente de la tecnología con fines sociales, se ha demostrado que no necesariamente se desenvuelven correctamente cuando hacen uso de la tecnología en el ámbito laboral o académico. De ahí que uno de los retos de las instituciones de educación superior sea darles los complementos de actitudes y habilidades transversales que les permitan convertir la información en conocimiento y utilizarla de forma ética, para que así, al incorporarse al mundo laboral, puedan tener un impacto positivo en las organizaciones y su entorno. El complemento que las competencias transversales ofrece a las competencias digitales tiene el potencial de ser el diferencial que las organizaciones busquen y valoren 
en las personas recién egresadas, y dicha transversalidad puede impulsar el desarrollo educativo y el progreso social del país.

Para ello, resulta fundamental promover el uso de modelos educativos centrados en el aprendizaje, con programas multimodales que coadyuven a la resolución de problemas nacionales y locales, por medio del pensamiento complejo, el emprendimiento y la innovación. Así como incorporar nuevos contenidos curriculares transversales que aseguren al estudiantado la adquisición de conocimientos útiles para la inserción al mundo profesional, ya que la pertinencia de estos permitirá entablar conexiones entre diferentes tipos de conocimiento y promover aprendizajes significativos de largo plazo. Esto destaca la importancia de fortalecer la cooperación entre las instituciones de educación superior, el Estado, la sociedad y el sector empresarial, por medio de proyectos, programas y servicios que impulsen el desarrollo del sector productivo, el fortalecimiento de la formación profesional y aseguren la empleabilidad de los grupos egresados universitarios. Solo así la educación superior en el México del siglo XXI estará en condiciones de consolidar las estrategias que le permitan asegurar la calidad educativa, la pertinencia, responsabilidad social, la equidad y la inclusión.

Para concluir, es importante señalar que las competencias adecuadas para México en el entorno digital tendrán que tomar como base la realidad del país y, a su vez, adaptarse a las exigencias de la política educativa global impulsada por organismos internacionales como la Organización Mundial del Comercio (OMC). Por tanto, además, resulta evidente la necesidad y pertinencia de la investigación educativa, que contribuya al análisis y permita al país mantenerse a la vanguardia con un enfoque prospectivo. De esta forma, el resultado de la vinculación entre las instituciones de educación superior y su entorno aportarán en el largo plazo las competencias específicas que se necesitan, ya que la transferencia de conocimientos y habilidades tienen que impactar al desarrollo local y global. En resumen, se puede afirmar que la inversión en la calidad académica adaptada a las necesidades de la era digital abre el camino al desarrollo y a una sociedad más competitiva e inclusiva con más oportunidades. 


\section{Referencias}

Aguirre, J. (2010). Filosofia y ciencia de la educación: Relación constante entre filosofia y educación. Buenos Aires: Editorial Brujas.

Asociación Mexicana de Internet. (2014). Estudio sobre los hábitos de los usuarios de internet en México 2014. Recuperado de https://www.amipci.org.mx/estudios/habitos_de_internet/ Estudio_Habitos_del_Internauta_Mexicano_2014_V_M̄MD.pdf

Almada, A. I. (2000). Formación de los recursos humanos y competencia laboral. Boletín cinterfor, 149, 1-14. Recuperado de file:///C:/Users/UQROO/Downloads/formacion_recursos_humanos_competencia_laboral_ibarra.pdf

Altarejos, C. y Naval, F. (2011). Filosofía de la educación (3a. ed.) Madrid: EUNSA.

Area, M. (2008). Innovación pedagógica con TIC y el desarrollo de las competencias informacionales y digitales. Investigación en la Escuela, 64, 5-18. Recuperado de http://manarea.webs.ull.es/ articulos/art16_investigacionescuela.pdf

Area, M. (2010). ¿ Por qué formar en competencias informacionales $\mathrm{y}$ digitales en la educación superior? RUSC. Universities and Knowledge Society Journal, 7(2), 6. Recuperado de file://C:/ Users/UQROO/Downloads/Dialnet-PorQueFormarEnCompetenciasInformacionalesYDigitale-3912752\%20(1).pdf

Cano, E. (2008). La evaluación por competencias en la educación superior. Profesorado: revista de currículum y formación del profesorado, 12(3), 11. Recuperado de https://www.ugr.es/ recfpro/ rev123COL1.pdf

Cazares, L y Cuevas, J. (2007). Planeación y evaluación basadas en competencias, México: Trillas

Consejo Nacional de Normalización y Certificación de Competencias Laborales. (2016) Consejo Nacional de Normalización y Certificación de Competencias Laborales. Recuperado de http://www.conocer.gob.mx/

DelaSelva,A.(2015). Losnuevos rostros dela desigualdaden elsigloXXI: La brecha digital. Revista mexicana de ciencias políticas y sociales, 60 (223), 265-285. Recuperado de: http://www.scielo.org.mx/ scielo.php?pid=S0185-19182015000100010\&script $=$ sci_arttext

Delors, J. (2013). Los cuatro pilares de la educación, Informe para la Unesco sobre Educación Superior. Revista Galileo, 23, 103-110. 
Recuperado de http://www.ucuenca.ec/ojs/index.php/galileo/ article/view/169

Denyer, M., Poulain, R., y Vanloubbeeck, G. (2004). Les compétences: où en est-on?: l'application du décret" Missions. En Communauté française de Belgique. Bruxelles: De Boeck.

Díaz, R. y Arancibia, V. (2011). Enfoque de las competencias laborales: Historia, definiciones y generación de un modelo de competencias para las organizaciones y las personas. Revista Psykhe, 11(2), 207- 214. Recuperado de file://C:/Users/UQROO/Desktop/1\%20 Doctos\%20Qroo/5\%20Investigaci\%C3\%B3n/2016/investigaci\%-

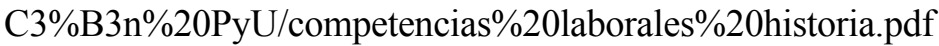

Díaz-Barriga (2010). Los profesores ante las innovaciones curriculares. Revista Iberoamericana de Educación Superior, 1(1), 37-57. Recuperado de https://ries.universia.net/article/view/32/1304

Didou, S. (2014). La UNESCO y la educación superior, 2014-2017: Aportes de la Reunión de Cátedras UNESCO sobre la educación superior, las TIC en la educación y los profesores. Recuperado de http://www.unesco.org/new/fileadmin/MULTIMEDIA/HQ/ ED/pdf/UNESCO-summary-report-chairs-2014-1.pdf

Dirección General de Educación Superior para Profesionales de la Educación (2016). Enfoque centrado en competencias. Recuperado de http://www.dgespe.sep.gob.mx/reforma_curricular/planes/ lepri/plan_de_estudios/enfoque_centrado_competencias

Real Academia Española. (2014). Definición de competencia. Recuperado de http://dle.rae.es/?id=A0fanvT|A0gTnnL

Ferrari, A. (2012). Digital Competence in Practice: an analysis of Frameworks. Seville: JRC IPTS.

Ferrater, M. J. (2003). Diccionario de Filosofía (Tomo II). Barcelona: Editorial Ariel.

Fromm, E. (2012). The sane society. London: Routledge.

González, J., Wagenaar, R., y Beneitone, P. (2004). Tuning-América Latina: Un proyecto de las universidades. Revista iberoamericana de educación, 35(1), 151-164. Recuperado de https://www.researchgate.net/profile/Robert_Wagenaar2/publication/28078676_TuningAmrica_Latina_un_proyecto_de_las_ universidades/links/0deec53c579b1998b4000000.pdf

World Economic Forum. (2016). Estos países enfrentan el mayor déficit de habilidades. Recuperado de https://www.weforum. 
org/es/agenda/2016/09/estos-paises-enfrentan-el-mayor-deficit-de-habilidades?utm_content $=$ buffer7b739\&utm_medium $=$ social\&utm source $=$ facebook.com\&utm campaign= $=$ buffer

Instituto Mexicano para la competitividad. (2014). Las reformas y los Estados. La responsabilidad de las entidades en el éxito de los cambios estructurales. Índice de Competitividad Estatal 2014. Recuperado de http://imco.org.mx/indices/\#!/ competitividad_estatal_2014/resultados/generales

Kezar, A. (2001). Understanding and facilitating organizational change in the 21 st century. ASHE-ERIC higher education report, 28(4), 1-162. Recuperado de http://onlinelibrary.wiley.com/ doi/10.1002/aehe.2804/abstract

Naismith, L., Sharples, M., Vavoula, G. y Lonsdale, P. (2004). Literature review in mobile technologies and learning. Futurelab Series,11, 1- 42. Recuperado de http://telearn.archives-ouvertes.fr/ docs/00/19/01/43/PDF/Naismith_2004.pdf

Organización para la Cooperación y Desarrollo Económico. (2012). Programa para la evaluación internacional de alumnos (PISA)Resultados. Recuperado de https://www.oecd.org/pisa/keyfindings/PISA-2012-results-mexico-ESP.pdf

Organización para la Cooperación y el Desarrollo Económicos. (2016). Definition and Selection of Competencies (DeSeCo). Recuperado de http://www.oecd.org/edu/skills-beyond-school/definitionandselectionofcompetenciesdeseco.htm

O’Malley, C., Vavoula, G., Glew, J., Taylor, J., Sharples, M. y Lefrere, P. (2003).Guidelines for learning/teaching/tutoring in a mobile environment. MOBIlearn, 4(1), 2-57 Recuperado de: http:// www.mobilearn.org/download/results/guidelines.pdf

Parametría. (2016). Peña y Mancera, con los niveles de aprobación más bajos de los últimos tres sexenios. Investigación estratégica, análisis de opinión y mercado. Recuperado de http://www.parametria.com.mx/DetalleParMedios.php?PM=1154

Peña, E. (2014). Las reformas transformadoras de México. Revista de Administración Pública, 49(1), 29. Recuperado de www.inap.org.mx.

Presidencia de la Republica. (2012). Plan Nacional de Desarrollo 2012-2018. Recuperado de http://pnd.gob.mx/

Presidencia de la República. (2015). Reformas. Recuperado de http:// www.gob.mx/presidencia/archivo/reformas 
Quintero, A. (2016). Renuncia de Peña. ¿Ya lo pensaste bien? Nexos. Recuperado de http://www.nexos.com.mx/?p=29618

Ramírez, M. S. (2012). Modelos y estrategias de enseñanza para ambientes innovadores. Monterrey, México: Editorial digital del Tecnológico de Monterrey.

Rodrigues, P., Peralta, M., y Nunes, C. (2011). Avaliação das aprendizagens no ensino superior: percepção de estudantes do Instituto de Educação da Universidade de Lisboa. $23^{\circ}$ Coloquio Internacional de ADMEE-Europe. Recuperado de http://www.ie.ulisboa.pt/ pls/portal/docs/1/333275.PDF

Secretaria de Educación Pública. (2016). El modelo educativo 2016. El planteamiento pedagógico de la Reforma Educativa. Recuperado de https://www.gob.mx/cms/uploads/attachment/file/114501/ Modelo_Educativo_2016.pdf

Secretaría de Gobernación. (2013). Introducción y visión general del Plan Nacional de Desarrollo 2012-2018. Recuperado de http://www.dof.gob.mx/nota_detalle. php? codigo $=5299465 \&$ fecha $=20 / 05 / 2013$

Spencer, L. y Spencer, S. (1991) Evaluación de competencias en el trabajo: Modelos para un desempeño superior. Boston: McBer and Company.

Tobón, S. (2008). La formación basada en competencias en la educación superior: El enfoque complejo. Grupo Cife, 1-30. Recuperado de http://cmapspublic3.ihmc.us/rid=1LVT9TXFX-1VKC0TM-16YT/Formaci\%C3\%B3n\%20basada $\% 20$ en $\% 20$ competencias\%20(Sergio\%20Tob\%C3\%B3n).pdf

Universitat Oberta de Catalunya. (2013). ¿Son importantes las competencias transversales en un proceso de selección? Recuperado de https://www.youtube.com/watch? $\mathrm{v}=4 \mathrm{wWgXfAMesU}$

Videgaray, L. (2014). El camino para transformar a México: Las reformas estructurales a un año de gobierno. Revista de Administración Pública,49, (1), 54. Recuperado de www.inap.org.mx.

Von Schelling, F. (2005). Sistema del idealismo trascendental. Barcelona: Anthropos Editorial. 